\title{
Sensing, Understanding, and Shaping Social Behavior
}

\author{
Erez Shmueli*, Vivek Kumar Singh*, Bruno Lepri and Alex "Sandy" Pentland
}

\begin{abstract}
An ability to understand social systems through the aid of computational tools is central to the emerging field of Computational Social Systems. Such understanding can answer epistemological questions on human behavior in a data-driven manner, and provide prescriptive guidelines for persuading humans to undertake certain actions in real world social scenarios. The growing number of works in this sub-field has the potential to impact multiple walks of human life including health, wellness, productivity, mobility, transportation, education, shopping, and sustenance.

The contribution of this paper is two-fold. First, we provide a functional survey of recent advances in sensing, understanding, and shaping human behavior, focusing on real world behavior of users as measured using passive sensors. Second, we present a case study on how trust, an important building block of computational social systems, can be quantified, sensed, and applied to shape human behavior. Our findings suggest that: 1) trust can be operationalized and predicted via computational methods (passive sensing and network-analysis), and 2) trust has a significant impact on social persuasion; in fact, it was found to be significantly more effective than the closeness of ties in determining the amount of behavior change.
\end{abstract}

Index Terms-Social systems; Persuasive computing; Mobile sensing; Trust; Social influence.

\section{INTRODUCTION}

An ability to understand social systems through the aid of computational tools is central to the emerging field of Computational Social Systems. This paper discusses the recent advances in computational approaches to sense, understand and shape human behavior. We consider this topic to be of utmost importance because: 1) it can answer epistemological questions on human behavior in a data-driven manner, and 2) provide prescriptive guidelines for persuading humans to undertake certain actions in the real world social scenarios. The growth spurt in this sub-field has the potential to impact multiple walks of human life including health, wellness, productivity, mobility, transportation, education, shopping, and sustenance. For example, computational tools can be used to

* Indicates equal contribution.

- E. Shmueli, V.K. Singh and A.S. Pentland are with the Media Lab, Massachusetts Institute of Technology, Cambridge, MA USA. E-mail: \{shmueli, singhv, pentland\}@mit.edu.

- B. Lepri is with the Media Lab, Massachusetts Institute of Technology, Cambridge, MA USA and the Fondazione Bruno Kessler, Trento, Italy. E-mail: lepri@fbk.eu. understand the spread of diseases in social networks and devise mechanisms to curtail it. Similarly a datadriven approach can be used to understand which users have the highest persuasive ability in a community and incentivize them to promote healthier lifestyles.

The first aim of this paper is to provide a functional (and not exhaustive) survey of recent advances in sensing, understanding, and shaping human behavior. This work broadens the existing literature by adopting a singular perspective. It focuses on real world behavior of users as measured using passive sensors.

Our work has a different focus from the large array of recent work on understanding cyber-world social behavior e.g. [45], [38], [42]. Clearly, each of these approaches have advanced the state of the art in understanding user-behavior in online settings. However, multiple studies have shown that users' real world behavior (face-to-face interactions, mobility patterns, calls, life-style choices) varies dramatically from their cyber behavior: cyber identities are often adopted; and sometimes even in direct contrast with how users behave in the real world [84], [86]. In fact, some of the more recent 'cyber' studies have found that most influential peers even in cyber settings are those with whom the user interacts frequently in the real world [9].

Moreover, with the growth in mobile and quantified sensing, we expect an ever-increasing proportion of user data to come inscribed with real-world spatiotemporal coordinates and social networking platforms to become more and more attunded to real world face-to-face interactions. Recent adoption of 'Places' and 'Events' features on Facebook, a 'mobile-first' platform growth for Instagram, and 'real-world' social networking on Highlight, Banjo, Galncee are all early indicators of this transition. We expect these trends to continue and undergo exponential growth in the coming decade.

This work also differs in focus from the survey based approaches adopted in social psychology to understand human behavior. While theoretically sound, these surveys tend to be expensive and often unsuitable for long term longitudinal analysis. Further they tend to be subjective and suffer from perception 
bias [31]. However, as the field of computational social systems is still evolving, we are seeing multiple studies that combine surveys with passive sensing. Such studies are included in the work to highlight how survey based methods could potentially be augmented or replaced by passive sensing methods in the near future.

The second aim of this paper is to present a case study on how trust, an important building block of computational social systems, can be quantified, sensed, and applied to shape human behavior. Trust is a central component of social and economic interactions among humans [56]. Bruce Scheiner in his book 'Liars and outliers' has further argued the case that enabling trust is essential to the thriving of societies [79]. Using a rich and dense sampling of the lives of $100+$ participants living in a single community for a year [2], we demonstrate how: (1) trust can be operationalized through a questionnaire and trusted relationships be predicted using computational methods based on passive sensing (of calls, SMS and Bluetooth interactions) and network analysis, and (2) trust has a significant impact on social persuasion: it was found to be significantly more effective than the closeness of ties in determining the amount of behavior change.

The rest of the paper is organized as follows. Section 2 surveys the recent state of the art in sensing and understanding human behavior. Section 3 discusses how computational methods are being employed to aid behavior change in social settings. Section 4 describes our recent results on quantifying, predicting and operationalizing trust as factor for behavioral change and section 5 summarizes our contributions and presents an outlook for the emerging sub-field.

\section{Sensing and Understanding BehaV- IOR}

There is a rich emerging literature on understanding social behavior using sensors. The applications studied in this context include health, mobility, personality, dating, meetings, affect, wellness, interest, gaze, overspending, and political interests. Similarly the modalities/sensors employed include audio, video, GPS, accelerometers, gaze trackers, skin conductivity sensors, thermal sensing, call data records, and so on.

In this section we sample a non-exhaustive array of works which bring out the variety of works being pursued. In keeping with the focus of the paper, we do not consider purely cyber-behavior or purely surveydriven real-world behavior. Similarly we study individual behavior, social behavior and their interplay but leave out the pure community/network understanding literature. Further, wherever possible we focus on higher level derived features and behaviors rather than raw sensing via a modality. Lastly, the sensing and signal processing community has made significant progress at understanding different aspects of human biometric signals. Efforts like facerecognition [89], speaker recognition [13], and gaze tracking [60] are now reasonably matured and left out of active in-depth discussion.

To provide a functional summary of the state of the art, we organize the discussion into four categories based on two dimensions: personal versus interpersonal, and short-term versus long-term behavior as shown in table 1. Generally speaking, a lot more progress has been made on understanding personal and short-term behaviors but there has been a steady stream of research efforts focusing on other aspects in recent years.

As the primary focus of this paper is on social behavior we will specifically highlight scenarios where inter-personal (i.e. social) behavior has been found to be predictive of personal actions and behavior (e.g. in health, sickness, affect, emotions, and financial wellbeing).

\subsection{Personal + Short-term}

\subsubsection{Emotional states}

There exists a large body of literature in machine analysis of human emotions e.g. [72], [67]. Most of these works attempt to recognize a set of prototypic expressions of basic emotions like happiness and anger from either face images/video or speech signal. There has been a spurt of recent work which tries to use social signals as a proxy for human affect. Multiple efforts use phone usage data to predict daily positive and negative affective states [48] and daily happiness states [8].

\subsubsection{Actions, poses, and gestures}

Various user actions such as sitting, eating, drinking etc. have been very well studied in image and video processing literature [11], [95]. Recent advances in sensor modalities e.g. Kinect and ego-centric cameras have allowed such detections to happen at much higher accuracy and response rates [73], [87]. Similarly there exists a plethora of work on gesture and pose recognition [59], [81] using audio, visual, and related modalities. With the growth of wearable computing there are also recent trends on wearable gloves and body suits that have been used for such action recognition [93]. Since most of these actions are short term effects and often detected with high accuracy there has been little active interest in employing social sensing mechanisms for these types of actions and poses.

There is also an emerging growth in sensor modalities and the kind of measurements about human body possible via them. For example, there have been a growth of 'quantified-self' sensors that measure the distance and the steps walked, calories burnt, accelerometer, heart rate, body temperature, gaze, skin conductance, portable ECG scans, sugar levels, and 
so on. However these devices measure body signals rather than long term behavior and hence are left outside the main focus of this survey.

\subsection{Personal + Long term}

\subsubsection{Personality}

Personality is often described as the long term typology of innate human characteristics that influence multiple actions taken by an individual. Hence identifying it is of interest in multiple applications including healthcare, product recommendation, and financial well-being. A number of different theories have been defined to study personality. However the most commonly used approaches are the Big-5 [37] and the locus-of-control [70]. Both have been studied by multiple groups using different modalities. For example [6] describes a method for using short introduction videos created by users to identify aspects of their big-5 personality traits. Similarly [71] uses a combination of audio-visual features to identify both big-5 and locus-of-control personality traits.

Interestingly, multiple efforts have started exploring the use of social signals e.g. interactions with others via mobile phones to identify personality traits. For example, [25] and [16] describes methods that use the cell-phone usage characteristics (e.g. time taken to respond, incoming vs outgoing call ratio, number of sent/received SMS) to predict personalities. Instead, [83] shows that individual personality traits can be predicted using structural characteristics (centrality measures, triads, transitivity) extracted from the call and proximity networks obtained from mobile phone data.

\subsubsection{Health and wellness}

Multiple efforts have been combining quantified-self signals to create longer term reports of healthiness of individuals. For example, The UbiFit Garden [22], a joint project between Intel and the University of Washington, captured levels of physical activity and related this information to personal health.

Recently, multiple efforts have explored the use of social sensing to detect flu onset both at an individual and a community level. For example [53] and [29] show how phone based social interaction features (e.g. locations visited, people encountered) can be used to predict the onset of influenza. Similarly [19] presents a method for wi-fi based tracking of social interactions that can be used to detect the onset of contagious outbreaks. In fact they show how the social network structure can be used to identify certain 'hubs' whose monitoring allows for a much earlier and more accurate prediction of the onset of the outbreak. Again, [52] uses high-resolution data on face-to-face interactions in a pediatric hospital ward, obtained from wearable proximity sensors, for simulating the spread of a disease inside this community.

\subsubsection{Financial behavior}

There has been a small but active movement towards using spending and other financial logs of users to identify their spending behavior. For example, [44] analyzed the spending logs for a large population and found that individuals who tend to have a positive economic outlook tend to visit diverse geo-locations for spending.

There is growing evidence that an individual's financial parameters: both income and spending are closely related to the social behavior. For example [66] has found that more socially diverse individuals tend to have higher income. Similarly [82] has found that couple's spending behavior in terms of the propensity to explore diverse businesses, become loyal customers, and overspend can be predicted by analyzing their social behavior.

\subsection{Interpersonal + Short-term}

\subsubsection{Roles in meetings}

Understanding user roles in official meetings has generated significant research interest [35]. For example, [5] described a method for using audio features for detecting roles in meetings. Multiple efforts since then have employed audio-visual sensing to undertake more sophisticated analysis of meeting roles [28], [96], [58]. As summarized in [35], different research groups have focused on different aspects of this process, including interaction management, internal states, and relationships. While most of the research has been undertaken using audio and visual sensors, other sensors like socio-metric badges have also recently been applied for analyzing meeting behavior [64].

\subsubsection{Outcomes of short-term group interaction}

Pentland [69] argues that certain inter-personal signals are too costly to fake and hence give away subtle feedback on the performance of the other person in interpersonal situations. Such signals include gaze, interest level, dominance, as well as social features like conversational turn taking. There is growing evidence that such features can be used to predict salary negotiations, hiring interviews, and speed-dating conversations [24]. These works are based on vocal social signals including overall activity (the total amount of energy in the speech signals), influence (the statistical influence of one person on the speaking patterns of the others), consistency (stability of the speaking patterns of each person), and mimicry (the imitation between people involved in the interactions). Similarly, other works have used cellular phones equipped with proximity sensors and vocal activity detectors to undertake automatic analysis of everyday social interactions in groups of several tens of individuals [68], [31]. 


\subsection{Interpersonal + Long-term}

2.4.1 Community structure: closeness, trust, strength of ties, and friendship

The work on using passive sensing to understand friendships, social ties, and the ensuing community patterns was pioneered by Eagle and Pentland in their 'reality mining' work [31] in 2006. Since then, there have been multiple efforts that have shown how Bluetooth, infra-red, wi-fi, and GPS sensors logs can be used to infer social structure and closeness of ties [53], [46]. While bluetooth and infra-red sensing are typically used for indoor one-on-one interaction assessment, wi-fi and GPS logs are used for coarser (but often wider coverage) analysis of people's mobility and co-location patterns.

Our case study in this paper (detailed in section 4) pays particular attention to the role of trust in social ties. In particular we show how call, sms, and Bluetooth based social interaction signals can be used to predict trust in relationship and quantify its role in mediating persuasion.

\subsubsection{Organizational effectiveness}

People's social ties and flow of information and expertise are closely tied to their performance. While this is obvious already through short-term meeting scenarios, the real impact lies in understanding long-term longitudinal performance. [65] describes a method that uses wearable electronic badges capable of automatically measuring the amount of face-toface interaction, conversational time, physical proximity to other people, and physical activity levels in order to capture individual and collective patterns of behavior. Using on-body sensors in large groups of people for extended periods of time in naturalistic settings, they tried to identify, measure, and quantify social interactions, group behavior, and organizational dynamics. Using these automatic measurements, they were able to predict employees self-assessments of job satisfaction and their own perceptions of group interaction quality.

Similarly, in another effort [63] the authors found that the social interaction patterns between nurses could be used to predict the daily average length of stay (LOS) and number of delays for their patients.

\section{Shaping Behavior}

Most of the research on persuasive technologies is comprised under the umbrella of the term captology [32], which refers to the study of machines designed to influence people's attitudes and behaviors through persuasion and social influence but not through coercion. In particular, a number of applications have been recently designed and implemented to promote positive behaviors like eating healthy foods, reducing carbon footprint, stopping smoking habits, and so on. Automatic persuasive interventions could have a number of advantages: first of all, they are costefficient. Then, they can be delivered anytime and anywhere: for example, just when they can have the highest impact on the users. Studies conducted in public places showed that motivational messages delivered at the time of making a choice inspired individuals to take simple decisions to improve their health, such as choosing a staircase and not an escalator [39]. However, if we contrast persuasion in human-human interaction and persuasion in humanmachine interaction, we still have a limited capability of machines for real-time understanding of people's individual traits, activities and social dynamics. As a consequence, most of the current persuasive systems lack flexibility and cannot personalize and adapt their message to the broader context the target person(s) is (are) in.

As we described in Section 2, the automatic analysis of human behavior has been progressing in the last few years thanks to the shared awareness that computer systems can provide better and more appropriate services to people only if they can understand much more about their attitudes, preferences, personality, social relationships etc., as well as about what people are doing, the activities they have been engaged in the past, their routines and lifestyles. In our survey of persuasive systems we will focus mainly on systems that use information about behaviors obtained by passive sensing to induce behavioral changes on users.

Following the definition given by Oinas-Kukkonen and Harjumaa [62], we also characterize persuasive technologies looking at how they induce changes on behaviors: (i) through a direct interaction and resorting to a human-computer persuasion strategy or (ii) through a mediation and so resorting to computermediated persuasion strategy (see table 2). In the first case, the system plays an active role and uses interaction patterns similar to social communication to induce a change in the user. This approach assumes that the computing system have a strong interaction with the user. In the second case, the system behaves in an implicit way: it may purposely work as a trigger of an informational cascade or it may purposely build links among people or it may simply show the behavior of peers to induce mimicry. The first two examples are network interventions while the latter is a social comparison strategy. In general, the computermediated approach has the advantage of not requiring a strong and long interaction with a machine and to use the innate ability of humans to engage in relationships characterized by social influence, peer pressure, and mimicking.

\subsection{Human Computer Persuasion}

The Computers as Social Actors (CASA) paradigm have argued that people apply social rules to computers [76]. Following this paradigm, the captology 
researchers have explored the usage of computers as persuaders. When the computers are used as persuaders, often the designers assumes that the system is the focus of the attention of the user and he/she exploits the fact that computers invoke social responses from users (especially, but not only, when being endowed with anthropomorphic characteristics). In particular, the majority of these studies investigated the persuasiveness of a computer agent delivering a monologue. Stern et al. [1] compared the persuasiveness of a message delivered by recorded human speech to the same message delivered by synthesized speech. The human voice was perceived as more favorable, but no differences in persuasion were found.

Bickmore et al. [7] designed Laura, an animated conversational agent displayed on a wall-mounted touch screen display, who acts as a virtual exercise advisor. In their study, the participants had to negotiate with Laura the daily step count goal. The negotiation took into account the current day's number of steps and the step count history and tried to gradually move each participant to her/his final step count goal at the end of the study. In a more recent study, Schulman and Bickmore [80] described the effects of a computer agent designed to engage a user in a persuasive dialogue on attitudes about regular physical exercise. The outcomes of the study showed that participants had a significant increase in positive attitudes following the persuasive dialogue.

Even when no explicit effort is done by the designers to have machines being perceived as social entities, a large portion of the work in the field of persuasive systems have resorted to an active role of the computer and to a direct human-computer interaction.

For example, several persuasive systems use a goalsetting strategy [50] as key component for inducing behavioral changes. In Fish'n'Steps [49] each participant has assigned a step count goal for each week of the study. Then, the ability of the participants in reaching their daily step count goal is mapped to the state (growth and emotional state) of a virtual pet, a fish in fish tank. Similarly, Gasser et al. employed assigned goals in their study of lifestyle automated coaching to provide users with feedback about their physical activity and vegetable and fruit intake [34].

More recently, Consolvo et al. [23] designed UbiFit, a mobile application that shows to the users detailed information about their activity behavior and the goal-attainment status. UbiFit uses several sensors embedded in the Mobile Sensing Platform (MSP) [17] for inferring different type of activities (e.g. walking, cycling, running, etc.). The metaphor used for giving feedback is the one of a garden that blooms during the week as physical activities are performed. Different types of flowers represent the types of activities relevant for the American College of Sports Medicine (ACSM): Cardio (pink flowers), Strength (blue), Flex- ibility (white), and Walking (sunflowers).

Houston [21] and Chick Clique [88] adds to this goal-setting strategy a social dimension giving to the users the functionality of sharing their step counts and their progress toward daily step goals with other users. However, the role played by the system is still an active role.

\subsection{Computer Mediated Human-Human Persua- sion}

Recently, some studies suggested that social interactions can be used by machines to induce users' behavioral change without requiring an active role by the computer and a direct and exclusive interaction with the users. As we said, the advantages of using the computer as a mediator and not as direct persuader is mainly related to the possibility of using the behaviors of peers for inducing changes. Here, we survey systems using 2 different strategies based on a computer-mediated persuasion. The first one is a social comparison strategy where the role of system is to show information about peers behaviors to elicit indirectly in the user a better awareness of his own behavior or a tendency to mimic the behavior of others. The second one, instead, is based on network interventions whereby the role of the system may be to suggest a change in the network structure or to hint specific peer-to-peer interactions. In both the cases the computer makes use of people to induce changes on the user.

\subsubsection{Social Awareness}

Some recent works have attempted at devising systems that present members of working group with information about their social behavior, with the goal of modifying it and making it more conducive to efficient group dynamics [27], [43], and [85].

DiMicco et al. [27] have investigated several peripheral displays that visualize the amount of participation of a small working group member in terms of speaking activity, with the purpose of stimulating selfreflection and improving individual social behavior.

A similar approach with the same purpose was pursued by Sturm and colleagues [85] using a tabletop device as peripheral display to show not only the speaking time but also the gaze behavior of the participants. Their results are similar to the ones found by DiMicco et al:: less overlapping speech, but no effect on gaze behaviour. In both these studies, the information about the behaviour of each individual member was available to the whole group.

Kim et al. [43] designed the Meeting Mediator, a mobile system that detects social interactions and provides real-time feedback to enhance group collaboration and performance. Social interactions are captured using Sociometric Badges [65]. The badges can collect unbiased and richer data than traditional methods by 
sensing body movement, proximity to other badges, and speech characteristics such as speaking speed and tone of voice. By visualizing this data in real-time on the mobile phone of each user, the Meeting Mediator induces changes in group collaboration patterns. In particular, the results shows greater productivity and trust within geographically separated groups that are using the Meeting Mediator.

Another interesting study is the one conducted by Balaam et al. [4] in which a multi-user public display was used to enhance the interactional synchrony visualizing subtle feedback about users' behavior. The findings from this study suggest that social dynamics can be used by machines to support group behavior without requiring a direct and exclusive interaction with the users. In all these examples, the focus is specifically on human-human interaction rather than on explicit human-computer interaction.

\subsubsection{Network Interventions}

Several studies have highlighted the influence of social networks on individual behaviors [18], [90], and [91]. In a recent review published on Science, Valente [91] made an effort to systematize the research efforts on network interventions, namely purposeful efforts to leverage social networks and social network data to generate social influence, induce and accelerate behavioral change and/or pursue and achieve desirable outcomes among individuals, communities, organizations, etc. In particular, Valente identified four major classes of network interventions: (i) targeting the influential subjects and use them for spreading the desired behavior, (ii) segmenting and targeting groups with particular network characteristics, (iii) altering (or designing and building) the network structure, and (iv) inducing particular peer-to-peer interaction.

A recent study, conducted by our group [2], tested an experimental intervention for inducing changes in fitness-related physical activities and habits. The intervention was based on inducing particular peerto-peer interaction and, more specifically, on a social mechanism in which subjects were rewarded based on their peers performance rather than their own. The main idea behind this mechanism is to incentivize subjects to exert (positive or negative) pressure on peers (see [55] for a theoretical foundation of the mechanism). In reality, such peer pressure may take many forms other than incentivizing fitnessrelated physical activities. For instance, in an energy consumption scenario examples of positive pressure include giving energy-saving advices to a neighbor while examples of negative pressure include inducing guilt or shame [41]. As we said, in [2] the goal was to encourage people to increase their daily physical activity. The physical activity level was captured using the accelerometer sensors of the participants' mobile phones.
Each participant had assigned two peers: some participants had close friends as their peers while other participants had strangers as their peers.

The study tested three different conditions: (i) the "self-monitoring" control condition (or individual feedback), whereby participants were rewarded for their own increased physical activity, (ii) the "peerview" condition (or social comparison), whereby the participant was shown the buddies' physical activities levels, but she/he was still rewarded for his/her own activity level, and (iii) the "peer-reward" condition, in which the buddies instead received a reward proportional to the participants activity. This condition simulates a network intervention and, more precisely, the social mechanism based on inducing peer-to-peer interactions and peer pressure.

A more detailed explanation of this study is reported in Section 4.4 where we analyze in detail the role played by levels of closeness and trust on the efficacy of "peer-view" and "peer-reward" conditions.

In a work environment setting, Waber et al. [92] conducted a two-phase study to investigate the effects of strong relationships inside social groups and to investigate how to increase the strength of groups in the workplace. In the first phase of the study, the researchers measured the interactions between workers at the call centre of a large bank based in US. These interactions were measured using Sociometric Badges [65]. They found that the strength of an individual's social group is positively associated to the productivity. In the second phase of the study, the researchers showed that by giving breaks to employees at the same time they increased the strength of the social groups of an individual. Interestingly, the intervention had an effect not only on the strength of the individuals social networks but also on increasing the productivity of the individuals. This study could be considered another example of network intervention and in particular a good example of altering/building the network structure.

\section{Trust}

Trust is a central component of social and economic interactions among humans [54]. Trust is often linked with the functioning of individuals, organizations and societies, resulting, usually, in positive outcomes. For instance, an individual that has an extensive network with high levels of trust, may obtain dividends from collaboration with others, by increasing access to distinct resources [20]. In organizations, trust is seen as a catalyst for collaboration and coordination between individuals which have the potential to improve information diffusion and innovation [12], [74]. At the society level, high levels of trust impose social controls on political and economic institutions, thereby increasing accountability, productivity and effectiveness [74]. Scheiner further argued trust to be essential to the thriving of societies [79]. 
Despite its importance, a clear scientific definition of trust that is well accepted across disciplines is missing. To illustrate that, a recent study by Castaldo [14] pulled together 72 published definitions of trust from numerous academic disciplines. Following are a few commonly used definitions:

- "an individual may be said to have trust in the occurrence of an event if he expects its occurrence and his expectation leads to behavior which he perceives to have greater negative motivational consequences if the expectation is not confirmed than positive motivational consequences if it is confirmed" [26].

- "accepted vulnerability to another's possible but not expected ill will (or lack of good will) toward one" [3].

- "the willingness of a party to be vulnerable to the actions of another party based on the expectation that the other will perform a particular action important to the trustor, irrespective of the ability to monitor or control that other party" [56].

- "The extent to which a person is confident in, and willing to act on the basis of, the words, actions, and decisions of another" [57].

- "a state involving confident positive expectations about another's motives with respect to oneself in situations entailing risk" [47].

- "expectations, assumptions or beliefs about the likelihood that another's future actions will be beneficial, favorable or at least not detrimental" [77].

- "a psychological state comprising the intention to accept vulnerability based upon positive expectations of the intentions or behavior of another" [78].

The variety of trust definitions and the differences between them is described by Borum [10] as follows: "The science is improving, but there is still a sense that, as in the proverb, researchers are often trying to define an elephant, while only being able to touch a particular part of it.".

In this section we suggest a way to computationally operationalize trust, measure it via passive sensing, and quantify its effect on social persuasion.

\subsection{Datasets}

In this study, we use the Friends and Family dataset, first introduced by Aharony et al. [2]. The Friends and Family dataset is based on a yearlong study, which used the "Funf" mobile phone platform, surveys, receipts/credit card statement, and a Facebook application, to collect an immensely rich and dense dataset on the lives of the 130 participants (approximately 64 families). The participants were all members of a young-family residential living community at a major North American university. All members of the community were couples, in which at least one of the members was affiliated with the university. The community was composed of over 400 residents, approximately half of which had children. The community had many ties of friendship between its members

Each participant was equipped with an Androidbased mobile phone incorporating the Funf platform. This platform is essentially a passive sensing software explicitly designed for collecting mobile data, such as Bluetooth of nearby devices, call and SMS logs, GPS location and accelerometer. In this paper, we focus on the Call, SMS and Bluetooth logs.

In addition to the passive data collection, subjects completed surveys at regular intervals. Monthly surveys included questions about self perception of relationships, group affiliation, interactions, and also standard scales like the Big-Five personality test [40]. Daily surveys included questions like mood, sleep, and other activity logging. In this paper, we focus on a survey administered as part of the study in order to determine the closeness and trust relationships between participants.

As incentive, the participants were able to keep the phone at the end of the study, and were compensated extra for every out-of-routine task, such as filling out surveys. Moreover, participation in interventions or sub-experiments was completely optional.

Compared with previous social computing observatory studies (e.g. [61], [31], [30]), the Friends and Family community includes a more diverse subject pool and provides a unique perspective into a phase of life that has not been traditionally studied in the field of ubiquitous computing - married couples and young families.

The study undertaken was approved by the Institutional Review Board (IRB) and conducted under strict protocol guidelines. The protection of participant privacy and sensitive information was a key consideration. For example, data were linked to coded identifiers for participants and to not their real world personal identifiers. All human-readable text, such as phone numbers and text messages, was captured as hashed identifiers, and never saved in clear text. Collected data were physically secured and de-identified before being aggregated for analysis. Additionally, data collection was designed to be as unobtrusive to subjects daily life as possible.

\subsection{Operationalizing trust}

As previously pointed out, trust has no clear scientific definition common to different disciplines. Moreover, the meaning of "trusting someone in a relationship" is dependent on the context of the relationship. Indeed, a subject $X$ may trust a subject $Y$ in a professional context but she may not trust the same person for babysitting her children. For this reason, we designed a questionnaire, having a simple operative way for assessing the trust relationship between two individuals. The questionnaire aims at modeling three different 
dimensions of trust (i.e., health, finance and family) by asking the following questions:

1) Would you ask person $X$ for help in sickness?

2) Would you ask person $X$ for a hundred dollar loan?

3) Would you ask person $X$ for babysitting?

The rationale behind the choice of these questions is that they cover the three key ingredients of the daily life of our participants: health, finance, and family.

The questionnaire was administered to the participants of the Friends and Family study, at the beginning of the study, and their answers are utilized in the following subsections as a measure of trust.

\subsection{Predicting trust}

In this section, we demonstrate how the interaction between a pair of individuals, captured through sensors in their mobile phone devices, can be used to predict the trust relationship between them.

\subsubsection{Features and target variables}

We considered three modalities for user interactions: Calls, SMS and Bluetooth (BT) logs. Each of these interaction modalities captures a different mode of human interaction.

- BT logs: captured the physical co-location of participants. The BT logs were created by scanning for nearby devices every five minutes in order to provide an estimate of the face to face interactions happening between the participant and other individuals. Knowing the BT identifiers of each smartphone in the study, we could thus infer when two participants' phones were in proximity. The five minutes sampling frequency prevented draining the battery while achieving a high enough resolution to detect social interaction.

- Call logs: captured distant, synchronous interactions. Hence the interacting individuals could be at different locations but were communicating at the same time.

- SMS logs: captured the distant, asynchronous, textual interactions. Unlike calls, and face-to-face interaction, in this interaction mode the users could be separated in both space and time.

For each relationship $\left(u_{1}, u_{2}\right)$, between the two participants $u_{1}$ and $u_{2}$, in the Friends and Family dataset, we extracted six social-behavioral features from each one of the three interaction networks above (i.e., Call, SMS and BT):

1) The number of interactions between $u_{1}$ and $u_{2}$.

2) The total number of interactions for $u_{1}$.

3) The total number of interactions for $u_{2}$.

4) The betweeness centrality of the edge $\left(u_{1}, u_{2}\right)$.

5) The betweeness centrality of the node $u_{1}$.

6) The betweeness centrality of the node $u_{2}$.
Where the betweeness centrality of a node/edge is the number of shortest paths from all nodes in the network to all others that pass through that node/edge [33].

We also defined three YES/NO target variables, Health, Finance and Family, corresponding to the three trust domain questions that were described in Subsection 4.2. In addition, we defined a fourth variable, $A L L$, which had the value YES if at least one of the three other corresponding variables had the value YES, and NO otherwise.

\subsubsection{Preprocessing}

In order to reduce noise caused by participants with incomplete data, we performed two stages of filtering: (1) We retained only relationships for which both participants had at least one call, one SMS and one BT interactions during the period of the study and (2) We retained only relationships for which the three trust questions were filled. At the end of the two filtering stages, our dataset contained 916 instances (i.e., relationships with 24 social-behavioral features and 4 trust target variables).

Unsurprisingly, for each one of the trust domains, the number of trusted instances was significantly smaller than the numbers of untrusted instances. For example, for the Health variable, only 148 out of the 916 values were YES. Similarly, the Finance variable had only 83 YES values, the Family variable had only 136 YES values and the All variable had only 166 YES values.

\subsubsection{Experimental setup and results}

We turn now to investigate the predictive power of the social-behavioral mobile features at classifying trust variables.

We performed a total of 16 trust-level classification experiments. Each experiment consisted of using the features of one modality (Call, SMS, BT) or their combination $(A l l)$ to classify one trust variable (Health, Finance, Family and All).

For our experiments, we used the Weka software (version 3.68) [36] and applied the J48 classifier (based on C4.5 [75]) with the following default settings: use unpruned tree $(U)=$ false; Confidence threshold for pruning $(C)=0.25$; minimum number of instances per leaf $(M)=2$; use reduced error pruning $(R)=$ false; number of folds for reduced error pruning $(\mathrm{N})=3$; use binary splits only $(\mathrm{B})=$ false; do not perform subtree raising (S)=false; do not clean up after the tree has been built $(\mathrm{L})=$ false; Laplace smoothing for predicted probabilities $(\mathrm{A})=$ false; seed for random data shuffeling $(\mathrm{Q})=1$.

We employed a ten fold cross validation strategy. Hence, each experiment consisted of executing 10 classification tasks as follows. First, the whole dataset was divided into ten partitions. Then in each execution, one of the 10 partitions was used for testing and the 
remaining 9 partitions were used for training. Finally, the results of the 10 classification tasks were averaged.

Figure 1 shows the accuracy (i.e., the percentage of correctly classified instances) for each one of the 16 trust-level classification experiments. For comparison, we also include the accuracy of a simple ZeroR classifier which always return the majority class as prediction. Since the dataset is highly imbalanced, the accuracy of the ZeroR classifier is already high and ranges between $81.88 \%$ to $90.94 \%$. Nevertheless, the J48 with any one of the four sets of features outperforms the results of the ZeroR classifier. For example, when using the All set of features, the accuracy ranges between $93.89 \%$ and $95.04 \%$.

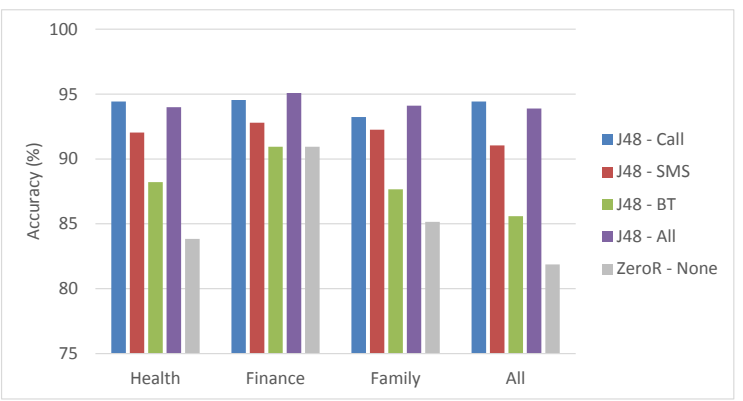

Fig. 1. Classification of trust-level: Accuracy results.

Since the dataset is highly imbalanced, we also applied two well-known measures for assessing the quality of classification in the case of imbalanced datasets.

Figures 2 and 3 show the Area Under the ROC Curve (AUC) measure and the F measure respectively, for each one of the 16 trust-level classification experiments. As can be seen in the figures, for each one of the 16 experiments, the results obtained by the J48 classifier are significantly better than those of the baseline ZeroR classifier.

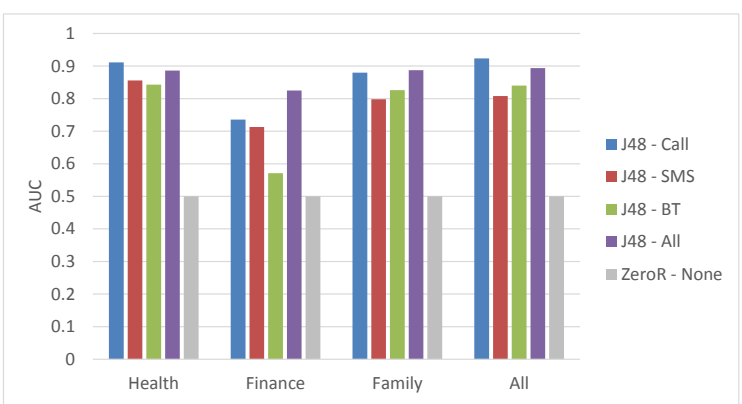

Fig. 2. Classification of trust-level: AUC results.

Figures 1-3 also illustrate the difference in prediction power between the different modalities. As can be seen across the three figures, the Call features seem to obtain better classification results than the SMS features, which in turn obtain better results than the $B T$ features. The results for the All features seem to

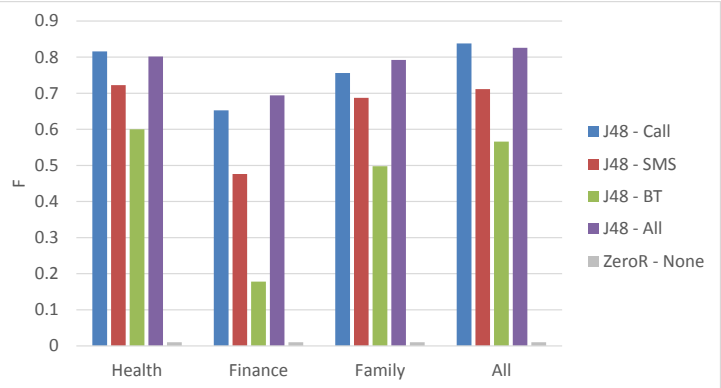

Fig. 3. Classification of trust-level: F Measure results.

be in par with those of the Call features (i.e., the SMS and $B T$ features do not seem to add prediction power on top of the Call features).

One explanation to the poor prediction ability of the BT features compared to that of the Call and $S M S$ features is the specific setting of the Friends and Family study. A lot of the face to face interactions between participants are likely to happen by chance, due to the small geographical region that they all live in. Call and SMS interactions on the contrary, require explicit actions by the interacting sides. These results corroborate the findings of [94] that networks derived from co-location and face-to-face conversations may be quite different.

Table 3 summarizes the results of the 16 experiments together with the results of the four ZeroR experiments.

\subsection{Social influence and trust}

Trust is integral to the idea of social influence - it is easier to influence or persuade someone who is trusting and people tend to mimic actions more often of those who they trust (e.g. [51]). In this section, we demonstrate the above idea using the FunFit experiment that was first described in [2]. In addition, we show, that under certain circumstances, trust plays a more significant role than closeness, when it comes to social influence.

FunFit is a fitness and physical activity experimental intervention conducted within the Friends and Family study population between October to December of 2010. The experiment was presented to participants as a wellness game to help them increase their daily activity levels. 108 subjects elected to participate and were divided into three experimental conditions:

1) Control - subjects saw only their own progress and were given reward based solely on their own activity.

2) Peer View - subjects were shown their own progress and the progress of two "buddies" also in the same experimental group, and were given reward based solely on their own activity.

3) Peer Reward - subjects were shown their own progress and the progress of two "buddies" 
also in the same experimental group, but their rewards depended solely on the performance of their buddies.

Out of the 108 subjects, eleven subjects were removed from the study pool due to prolonged technical issues that prevented reliable activity tracking, long durations of out of town travel, or dropping out of the longitudinal study entirely. The remaining 97 subjects were distributed among the three experimental groups as follows: 16 in the Control group, 40 in the Peer View group and 41 in the Peer Reward group.

During the initial 23 days of the experiment (Oct 5 - Oct 27), denoted as P1, the baseline activity levels of the subjects were collected. The actual intervention period was divided into two periods: Oct 28 - Nov 15, denoted as P2, and Nov 16 - Dec 5, denoted as P3. During these two intervention periods, the subjects were given feedback on their performance in the form of a monetary reward. The monetary reward was calculated as a function of the subject's activity and was divided according to the subject's experimental condition. The game was not designed as a competition, and every subject had the potential to earn the maximal reward (up to $\$ 5$ was allocated every three days). For further information on the FunFit experimental design, the reader is referred to [2].

The results in [2] suggest that there is a notion of a complex contagion [15] like effect related to preexisting social ties between participants. Our focus here is to zoom-in to these results and analyze how closeness and trust, as two different types of social ties, interplay in this contagion process.

In order to undertake this analysis, we first divided the subjects into two groups according to their trust level in their two Buddies: (1) None of the buddies is trusted and (2) at least one of the buddies is trusted. The trust level was determined according to our operative definition: a buddy is considered trusted if at least one of the three trust questions is answered YES. We then tested the influence of the trust level in buddies on the improvement in activity level as follows: (1) We calculated the average user activity level for each period and each group; (2) We calculated the improvement in activity level (measured in \%) from period P1 to period P3 for each group (similarly to [2] we focus on P3 since we want to look at longer-term adherence); and (3) We calculated the difference (delta) in improvement between the two groups. As shown in Table 4, in the Peer View condition, subjects that have at least one trusted buddy obtain additional $4.95 \%$ improvement in their activity level compared to subjects with 0 trusted buddies. On the Peer Reward condition, however, the improvement for subjects with at least one trusted buddy is inferior to that of subjects with 0 trusted buddies (13.39\% and $8.30 \%$ respectively).

Next, we divided the subjects into two groups according to their closeness level to their two Buddies: (1) None of the buddies is close and (2) at least one of the buddies is close. The closeness level that was reported in a survey as part of the Friends and Family dataset, was measured at a scale of 0 to 7 , and similarly to [2], we used a threshold of 3 to indicate a close friend. We then tested the influence of the closeness level in buddies on the improvement in activity level in the same way that we did it for the trust level. As shown in Table 5, in the Peer View condition, subjects that have at least one close buddy obtain roughly no additional improvement (i.e., $0.02 \%$ ) in their activity level compared to subjects with 0 close buddies. However, in the Peer Reward condition, the delta is high: subjects that have at least one close buddy obtain additional $9.33 \%$ improvement in their activity level compared to subjects with 0 close buddies.

Finally, comparing the results in tables 4 and 5 indicates that in the "Peer View" condition, the additional improvement that trusted buddies obtain is significantly higher than that of close buddies $(4.95 \%$ compared to $0.02 \%$ ). As for the Peer Reward condition, the additional improvement that the close buddies obtain is higher. One explanation for the different results in the two experimental conditions could be the following: When monetary social incentives are introduced, like in the Peer Reward condition, the closeness level between the subject and her buddies may play a more significant role than her trust in them. That could be a result of two factors: (1) Close buddies would feel more comfortable in contacting the subject more often and persuade her to improve her activity level and (2) The subject would care for her close buddies and would like them to earn more money, while she might not care for her trusted buddies in the same way. In the "Peer View" condition, on the other hand, monetary incentives are not present and the subject's improvement depends mostly on comparing herself to her buddies. In this case, she is more likely to mimic buddies that she trusts, and that's why we see the high delta in improvement. We would also like to emphasize that in many real-world scenarios, the Peer View setting is more applicable than the Peer Reward setting since it may work well even without monetary incentives.

For completeness, Table 6 illustrates the similarity and difference between the trust and closeness relationships in the FunFit experiment. As can be seen in the table, out of the 162 relationships (corresponding to the 81 subjects in the Peer View and Peer Reward groups), 118 are neither trusted nor close; 18 are both trusted and close; 5 are trusted but not close and 22 are close but not trusted.

\section{SummarY}

The first part of this paper surveys the recent advances in computational approaches to sense, understand and shape human behavior. We consider this 
TABLE 1

A summary of works on sensing and understanding behavior

\begin{tabular}{|l|l|l|}
\hline & Personal & Inter-personal \\
\hline Short-term & Emotional states [72], [67], [48], [8] & Roles in meetings [35], [28], [96], [58], [5], [64] \\
& Actions, poses, and gestures [59], [81], [73], [87], [59], [81], [93] & Outcomes of short-term group interactions [69] ,[68], [31], [25], [24] \\
\hline Long-term & $\begin{array}{l}\text { Personality [6],[71],[25], [83], [16] } \\
\text { Health and wellness [22], [53], [19], [52] } \\
\text { Financial behavior [44], [82], [66] }\end{array}$ & Organizational effectiveness [65], [63] \\
\hline
\end{tabular}

TABLE 2

A summary of works on shaping behavior

\begin{tabular}{|c|l|}
\hline Human-Computer Persuasion & Computer Mediated Human-Human Persuasion \\
\hline Conversational Agents [7], [80] & Social Awareness [27], [85], [43], [4] \\
Goal-setting [49], [34], [23], [21], [88] & Network Interventions [2], [55], [92] \\
\hline
\end{tabular}

TABLE 3

A summary of the trust classification results.

\begin{tabular}{|c|c|c|c|c|c|c|c|c|c|c|c|c|c|c|}
\hline Target & Features & Model & Instances & YES & NO & TP & FP & TN & FN & Precision & Recall & Accuracy & AUC & $\bar{F}$ \\
\hline Health & Call & J48 & 916 & 768 & 148 & 113 & 16 & 752 & 35 & 0.88 & 0.76 & 94.43 & 0.91 & 0.82 \\
\hline Health & SMS & $J 48$ & 916 & 768 & 148 & 95 & 20 & 748 & 53 & 0.83 & 0.64 & 92.03 & 0.86 & 0.72 \\
\hline Health & BT & $J 48$ & 916 & 768 & 148 & 81 & 41 & 727 & 67 & 0.66 & 0.55 & 88.21 & 0.84 & 0.60 \\
\hline Health & All & J48 & 916 & 768 & 148 & 111 & 18 & 750 & 37 & 0.86 & 0.75 & 94.00 & 0.89 & 0.80 \\
\hline Health & None & ZeroR & 916 & 768 & 148 & 0 & 0 & 768 & 148 & 0.00 & 0.00 & 83.84 & 0.50 & 0.00 \\
\hline Finance & Call & J48 & 916 & 833 & 83 & 47 & 14 & 819 & 36 & 0.77 & 0.57 & 94.54 & 0.74 & 0.65 \\
\hline Finance & SMS & $\mathrm{J} 48$ & 916 & 833 & 83 & 30 & 13 & 820 & 53 & 0.70 & 0.36 & 92.79 & 0.71 & 0.48 \\
\hline Finance & BT & J48 & 916 & 833 & 83 & 9 & 9 & 824 & 74 & 0.50 & 0.11 & 90.94 & 0.57 & 0.18 \\
\hline Finance & All & J48 & 916 & 833 & 83 & 51 & 13 & 820 & 32 & 0.80 & 0.61 & 95.09 & 0.83 & 0.69 \\
\hline Finance & None & ZeroR & 916 & 833 & 83 & 0 & 0 & 833 & 83 & 0.00 & 0.00 & 90.94 & 0.50 & 0.00 \\
\hline Family & "Call & J48 & 916 & 780 & 136 & 96 & 22 & 758 & 40 & 0.81 & 0.71 & 93.23 & 0.88 & 0.76 \\
\hline Family & SMS & J48 & 916 & \begin{tabular}{|l|}
780 \\
\end{tabular} & 136 & 78 & 13 & 767 & 58 & 0.86 & 0.57 & 92.25 & 0.80 & 0.69 \\
\hline Family & BT & $J 48$ & 916 & 780 & 136 & 56 & 33 & 747 & 80 & 0.63 & 0.41 & 87.66 & 0.83 & 0.50 \\
\hline Family & All & $J 48$ & 916 & 780 & 136 & 103 & 21 & 759 & 33 & 0.83 & 0.76 & 94.10 & 0.89 & 0.79 \\
\hline Family & None & ZeroR & 916 & \begin{tabular}{|l|}
780 \\
\end{tabular} & 136 & 0 & 0 & 780 & 136 & 0.00 & 0.00 & 85.15 & 0.50 & 0.00 \\
\hline All & Call & J48 & 916 & 750 & 166 & 132 & 17 & 733 & 34 & 0.89 & 0.80 & 94.43 & 0.92 & 0.84 \\
\hline All & SMS & J48 & 916 & 750 & 166 & 101 & 17 & 733 & 65 & 0.86 & 0.61 & 91.05 & 0.81 & 0.71 \\
\hline All & BT & $\mathrm{J} 48$ & 916 & 750 & 166 & 86 & 52 & 698 & 80 & 0.62 & 0.52 & 85.59 & 0.84 & 0.57 \\
\hline All & All & $\mathrm{J} 48$ & 916 & 750 & 166 & 133 & 23 & 727 & 33 & 0.85 & 0.80 & 93.89 & 0.89 & 0.83 \\
\hline All & None & ZeroR & 916 & 750 & 166 & 0 & 0 & 780 & 136 & 0.00 & 0.00 & 81.88 & 0.50 & 0.00 \\
\hline
\end{tabular}

TABLE 4

Activity level of participants in the FunFit experiment, grouped according to their trust level in buddies.

\begin{tabular}{|c|c|c|c|c|c|c|c|c|c|c|}
\hline \multirow{3}{*}{ Condition } & \multirow{3}{*}{ Period } & \multicolumn{8}{|c|}{ Trusted Buddies } & \multirow{3}{*}{ Delta } \\
\hline & & \multicolumn{4}{|c|}{0} & \multicolumn{4}{|c|}{ At least 1} & \\
\hline & & $\mathbf{N}$ & Mean & STD & $\%$ Improvement & $\mathbf{N}$ & Mean & STD & $\%$ Improvement & \\
\hline \multirow{2}{*}{ Peer View } & P1 & 31 & 1.331 & 0.481 & \multirow{2}{*}{$-0.62 \%$} & 8 & 1.408 & 0.396 & \multirow{2}{*}{$4.33 \%$} & \multirow{2}{*}{$4.95 \%$} \\
\hline & P3 & 31 & 1.323 & 0.420 & & 9 & 1.469 & 0.455 & & \\
\hline \multirow{2}{*}{ Peer Reward } & P1 & 33 & 1.223 & 0.503 & \multirow{2}{*}{$13.39 \%$} & 7 & 1.193 & 0.475 & \multirow{2}{*}{$8.30 \%$} & \multirow{2}{*}{$-5.09 \%$} \\
\hline & P3 & 34 & 1.387 & 0.413 & & 7 & 1.292 & 1.292 & & \\
\hline
\end{tabular}


TABLE 5

Activity level of participants in the FunFit experiment, grouped according to their closeness level to buddies.

\begin{tabular}{|c|c|c|c|c|c|c|c|c|c|c|}
\hline \multirow{3}{*}{ Condition } & \multirow{3}{*}{ Period } & \multicolumn{8}{|c|}{ Close Buddies } & \multirow{3}{*}{ Delta } \\
\hline & & \multicolumn{4}{|c|}{0} & \multicolumn{4}{|c|}{ At least 1} & \\
\hline & & $\mathbf{N}$ & Mean & STD & \% Improvement & $\mathbf{N}$ & Mean & STD & $\%$ Improvement & \\
\hline \multirow{2}{*}{ Peer View } & P1 & 25 & 1.346 & 0.413 & \multirow{2}{*}{$0.65 \%$} & 14 & 1.349 & 0.553 & \multirow{2}{*}{$0.67 \%$} & \multirow{2}{*}{$0.02 \%$} \\
\hline & $\mathrm{P} 2+\mathrm{P} 3$ & 25 & 1.355 & 0.420 & & 15 & 1.358 & 0.454 & & \\
\hline \multirow{2}{*}{ Peer Reward } & P1 & 23 & 1.259 & 0.523 & \multirow{2}{*}{$8.77 \%$} & 17 & 1.163 & 0.458 & \multirow{2}{*}{$18.10 \%$} & \multirow{2}{*}{$9.33 \%$} \\
\hline & $\mathrm{P} 2+\mathrm{P} 3$ & 24 & 1.369 & 0.412 & & 17 & 1.373 & 1.373 & & \\
\hline
\end{tabular}

TABLE 6

Contrasting trust and closeness relationships.

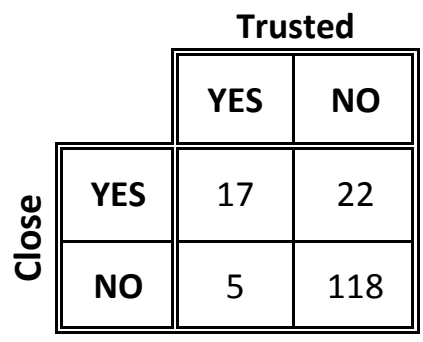

topic to be extremely timely as such sensor driven understanding of people, organizations, and societies at scale is now becoming possible for the first time. The avalanche of data coming from the emerging social computational systems will soon allow us to verify and refine multiple theories in organizational communication, information flow, social psychology and so on, and also create practical solutions that use this knowledge to persuade users to take actions beneficial for individual and societal good. Together, these approaches will impact multiple aspects of human life including health and wellness, civic infrastructure, emergency response, economic wellbeing, quality of life, and organizational effectiveness.

Our survey in this paper has a specific focus on real world behaviors of users as measured using passive sensors such as cameras, microphones, wearable sensors and mobile phones. In the last few years, the automatic analysis of human behavior from passive sensor data has been appliied to multiple domains including health and wellness, emotional states, roles in meetings, outcomes of short-term interactions, organizational effectiveness and so on. Although a lot more progress has been made on understanding personal and short-term behaviors, there has been a steady stream of research efforts focusing on other aspects in recent years.

With regard to behavior shaping, captology studies have traditionally focused on the use of computers as active persuaders. Recently, multiple studies have suggested that (computer-mediated) social interactions may work better than computers-as-persuaders in many scenarios. One possible explanation for this is that humans are much more socially aware, adaptive, and persuasive than computers in most practical situations. Further, they also create opportunities for 'behavior mimicing' in such systems.

In the second part of this paper, we have presented a case study on how trust, an important building block of computational social systems, can be quantified, sensed, and applied to shape human behavior. Using a rich and dense sampling of the lives of $100+$ participants living in a single community for a year [2], we have demonstrated that: (1) trust can be operationalized through a questionnaire and trusted relationships be predicted using computational methods based on passive sensing (calls, SMS and Bluetooth interactions) and network analysis, and (2) trust has a significant impact on social persuasion: it was found to be significantly more effective than the closeness of ties in determining the amount of behavior change.

In this paper, we validate our findings about trust and social persuasion based on a fitness and physical activity intervention study. However, the relationship between trust and social persuasion is a key element in a variety of scenarios. In the future, we plan to validate our findings in additional real-world settings such as energy consumption, mobility, sustainable living, and economic well-being.

\section{REFERENCES}

[1] S. E. S. aand John W. Mullennix, C. lynn Dyson, and S. J. Wilson. The persuasiveness of synthetic speech versus human speech. Human Factors, 41(4):588-595, 1999.

[2] N. Aharony, W. Pan, C. Ip, I. Khayal, and A. Pentland. Social fmri: Investigating and shaping social mechanisms in the real world. Pervasive and Mobile Computing, 7(6):643-659, 2011

[3] A. Baier. Trust and antitrust. Ethics, 96(2):231-260, 1986.

[4] M. Balaam, G. Fitzpatrick, J. Good, and E. Harris. Enhancing interactional synchrony with an ambient display. In CHI, pages 867-876, 2011.

[5] S. Banerjee and A. I. Rudnicky. Using simple speech-based features to detect the state of a meeting and the roles of the meeting participants. 2004.

[6] L. M. Batrinca, N. Mana, B. Lepri, F. Pianesi, and N. Sebe. Please, tell me about yourself: automatic personality assessment using short self-presentations. In Proceedings of the 13th international conference on multimodal interfaces, pages 255-262. ACM, 2011.

[7] T. W. Bickmore, L. Caruso, K. Clough-Gorr, and T. Heeren. 'it's just like you talk to a friend' relational agents for older adults. Interacting with Computers, 17(6):711-735, 2005.

[8] A. Bogomolov, B. Lepri, and F. Pianesi. Happiness recognition from mobile phone data. ASE/IEEE Social Computing conference, 2013. 
[9] R. M. Bond, C. J. Fariss, J. J. Jones, A. D. Kramer, C. Marlow J. E. Settle, and J. H. Fowler. A 61-million-person experiment in social influence and political mobilization. Nature, 489(7415):295-298, 2012.

[10] R. Borum. The science of interpersonal trust. 2010.

[11] M. Brand, N. Oliver, and A. Pentland. Coupled hidden markov models for complex action recognition. In Computer Vision and Pattern Recognition, 1997. Proceedings., 1997 IEEE Computer Society Conference on, pages 994-999. IEEE, 1997.

[12] R. S. Burt. The network structure of social capital. Research in organizational behavior, 22:345-423, 2000.

[13] J. P. Campbell Jr. Speaker recognition: A tutorial. Proceedings of the IEEE, 85(9):1437-1462, 1997.

[14] S. Castaldo. Trust in market relationships. Edward Elgar Publishing, 2007.

[15] D. Centola and M. Macy. Complex contagions and the weakness of long ties1. American Journal of Sociology, 113(3):702-734, 2007.

[16] G. Chittaranjan, J. Blom, and D. Gatica-Perez. Mining largescale smartphone data for personality studies. Personal and Ubiquitous Computing, 17(3):433-450, 2013.

[17] T. Choudhury, G. Borriello, S. Consolvo, D. Hähnel, B. L. Harrison, B. Hemingway, J. Hightower, P. V. Klasnja, K. Koscher, A. LaMarca, J. A. Landay, L. LeGrand, J. Lester, A. Rahimi, A. D. Rea, and D. Wyatt. The mobile sensing platform An embedded activity recognition system. IEEE Pervasive Computing, 7(2):32-41, 2008.

[18] N. A. Christakis and J. H. Fowler. The spread of obesity in a large social network over 32 years. New England Journal of Medicine, 357(4):370-379, 2007.

[19] N. A. Christakis and J. H. Fowler. Social network sensors for early detection of contagious outbreaks. PloS one, 5(9):e12948, 2010.

[20] J. S. Coleman. Social capital in the creation of human capital. American journal of sociology, pages S95-S120, 1988.

[21] S. Consolvo, K. Everitt, I. E. Smith, and J. A. Landay. Design requirements for technologies that encourage physical activity. In CHI, pages 457-466, 2006.

[22] S. Consolvo, D. W. McDonald, T. Toscos, M. Y. Chen, J. Froehlich, B. Harrison, P. Klasnja, A. LaMarca, L. LeGrand, R. Libby, et al. Activity sensing in the wild: a field trial of ubifit garden. In Proceedings of the SIGCHI Conference on Human Factors in Computing Systems, pages 1797-1806. ACM, 2008.

[23] S. Consolvo, D. W. McDonald, T. Toscos, M. Y. Chen, J. Froehlich, B. L. Harrison, P. V. Klasnja, A. LaMarca, L. LeGrand, R. Libby, I. E. Smith, and J. A. Landay. Activity sensing in the wild: a field trial of ubifit garden. In $\mathrm{CHI}$, pages 1797-1806, 2008.

[24] J. R. Curhan and A. Pentland. Thin slices of negotiation: Predicting outcomes from conversational dynamics within the first 5 minutes. Journal of Applied Psychology, 92(3):802, 2007.

[25] Y.-A. de Montjoye, J. Quoidbach, F. Robic, and A. S. Pentland. Predicting personality using novel mobile phone-based metrics. In Social Computing, Behavioral-Cultural Modeling and Prediction, pages 48-55. Springer, 2013.

[26] M. Deutsch. The effect of motivational orientation upon trust and suspicion. Human relations, 13(2):123-139, 1960.

[27] J. M. DiMicco, K. J. Hollenbach, A. Pandolfo, and W. Bender. The impact of increased awareness while face-to-face. HumanComputer Interaction, 22(1-2):47-96, 2007.

[28] W. Dong, B. Lepri, A. Cappelletti, A. S. Pentland, F. Pianesi, and $\mathrm{M}$. Zancanaro. Using the influence model to recognize functional roles in meetings. In Proceedings of the 9th international conference on Multimodal interfaces, pages 271-278. ACM, 2007.

[29] W. Dong, A. Pentland, and K. A. Heller. Graph-coupled hmms for modeling the spread of infection. In UAI, pages 227-236, 2012.

[30] N. Eagle, M. Macy, and R. Claxton. Network diversity and economic development. Science, 328(5981):1029-1031, 2010.

[31] N. Eagle and A. Pentland. Reality mining: sensing complex social systems. Personal and ubiquitous computing, 10(4):255268, 2006.

[32] B. Fogg. Persuasive technology: Using computers to change what we think and do. Morgan Kaufmann, 1999.

[33] L. C. Freeman. A set of measures of centrality based on betweenness. Sociometry, pages 35-41, 1977.
[34] R. Gasser, D. Brodbeck, M. Degen, J. Luthiger, R. Wyss, and S. Reichlin. Persuasiveness of a mobile lifestyle coaching application using social facilitation. In Proceedings of the First International Conference on Persuasive Technology for Human Wellbeing, PERSUASIVE'06, pages 27-38. Springer-Verlag, 2006.

[35] D. Gatica-Perez. Automatic nonverbal analysis of social interaction in small groups: A review. Image and Vision Computing, 27(12):1775-1787, 2009.

[36] M. Hall, E. Frank, G. Holmes, B. Pfahringer, P. Reutemann, and I. H. Witten. The weka data mining software: an update. ACM SIGKDD Explorations Newsletter, 11(1):10-18, 2009.

[37] J.-D. Haynes and G. Rees. Decoding mental states from brain activity in humans. Nature Reviews Neuroscience, 7(7):523-534, 2006.

[38] B. Huberman, D. Romero, and F. Wu. Social networks that matter: Twitter under the microscope. Available at SSRN 1313405, 2008.

[39] S. Intille. A new research challenge: persuasive technology to motivate healthy aging. IEEE Transactions on Information Technology in Biomedicine, 8(3):235-237, 2004.

[40] O. P. John and S. Srivastava. The big five trait taxonomy: History, measurement, and theoretical perspectives. Handbook of personality: Theory and research, 2:102-138, 1999.

[41] E. Kandel and E. P. Lazear. Peer pressure and partnerships. Journal of Political Economy, 100(4):pp. 801-817, 1992.

[42] D. Kempe, J. Kleinberg, and E. Tardos. Maximizing the spread of influence through a social network. In Proceedings of the ninth ACM SIGKDD international conference on Knowledge discovery and data mining, pages 137-146. ACM, 2003.

[43] T. Kim, A. Chang, L. Holland, and A. Pentland. Meeting mediator: enhancing group collaborationusing sociometric feedback. In CSCW, pages 457-466, 2008.

[44] C. Krumme, A. Llorente, M. Cebrian, et al. The predictability of consumer visitation patterns. Scientific reports, 3, 2013.

[45] R. Kumar, J. Novak, and A. Tomkins. Structure and evolution of online social networks. In Link Mining: Models, Algorithms, and Applications, pages 337-357. Springer, 2010.

[46] N. D. Lane, E. Miluzzo, H. Lu, D. Peebles, T. Choudhury, and A. T. Campbell. A survey of mobile phone sensing. Communications Magazine, IEEE, 48(9):140-150, 2010.

[47] R. J. Lewicki and B. B. Bunker. Trust in relationships: A model of development and decline. Jossey-Bass, 1995.

[48] R. LiKamWa, Y. Liu, N. D. Lane, and L. Zhong. Moodscope: building a mood sensor from smartphone usage patterns. In MobiSys, pages 389-402, 2013.

[49] J. J. Lin, L. Mamykina, S. Lindtner, G. Delajoux, and H. B. Strub. Fish'n'steps: Encouraging physical activity with an interactive computer game. In Proceedings of the 8th International Conference on Ubiquitous Computing, UbiComp'06, pages 261278, Berlin, Heidelberg, 2006. Springer-Verlag.

[50] E. A. Locke and G. P. Latham. Building a practically useful theory of goal setting and task motivation - a 35-year odyssey, 2002.

[51] R. López-Pérez. Reciprocity, social influence, and trust. 2005.

[52] A. Machens, F. Gesualdo, C. Rizzo, A. E. Tozzi, A. Barrat, and C. Cattuto. An infectious disease model on empirical networks of human contact: bridging the gap between dynamic network data and contact matrices. BMC Infectious Diseases, 13, Apr 2013.

[53] A. Madan, M. Cebrian, S. Moturu, K. Farrahi, and S. Pentland. Sensing the health stateof a community. 2012

[54] M. L. Manapat, M. A. Nowak, and D. G. Rand. Information, irrationality, and the evolution of trust. Journal of Economic Behavior \& Organization, 2012

[55] A. Mani, I. Rahwan, and A. Pentland. Inducing peer pressure to promote cooperation. Scientific Reports, 2013.

[56] R. C. Mayer, J. H. Davis, and F. D. Schoorman. An integrative model of organizational trust. Academy of management review, 20(3):709-734, 1995.

[57] D. J. McAllister. Affect-and cognition-based trust as foundations for interpersonal cooperation in organizations. Academy of management journal, 38(1):24-59, 1995.

[58] L. McCowan, D. Gatica-Perez, S. Bengio, G. Lathoud, M. Barnard, and D. Zhang. Automatic analysis of multimodal group actions in meetings. Pattern Analysis and Machine Intelligence, IEEE Transactions on, 27(3):305-317, 2005. 
[59] S. Mitra and T. Acharya. Gesture recognition: A survey. Systems, Man, and Cybernetics, Part C: Applications and Reviews, IEEE Transactions on, 37(3):311-324, 2007.

[60] C. H. Morimoto and M. R. Mimica. Eye gaze tracking techniques for interactive applications. Computer Vision and Image Understanding, 98(1):4-24, 2005.

[61] J. M. Norvilitis, M. M. Merwin, T. M. Osberg, P. V. Roehling, P. Young, and M. M. Kamas. Personality factors, money attitudes, financial knowledge, and credit-card debt in college students1. Journal of Applied Social Psychology, 36(6):1395-1413, 2006.

[62] H. Oinas-Kukkonen and M. Harjumaa. A systematic framework for designing and evaluating persuasive systems. In Persuasive Technology, volume 5033 of Lecture Notes in Computer Science, pages 164-176. Springer Berlin Heidelberg, 2008.

[63] D. O. Olguín, P. A. Gloor, and A. Pentland. Wearable sensors for pervasive healthcare management. In Pervasive Computing Technologies for Healthcare, 2009. PervasiveHealth 2009. 3rd International Conference on, pages 1-4. IEEE, 2009.

[64] D. O. Olguin, P. A. Gloor, and A. S. Pentland. Capturing individual and group behavior with wearable sensors. In Proceedings of the 2009 aaai spring symposium on human behavior modeling, SSS, volume 9, 2009.

[65] D. O. Olguín, B. N. Waber, T. Kim, A. Mohan, K. Ara, and A. Pentland. Sensible organizations: Technology and methodology for automatically measuring organizational behavior. Systems, Man, and Cybernetics, Part B: Cybernetics, IEEE Transactions on, 39(1):43-55, 2009.

[66] W. Pan, N. Aharony, and A. Pentland. Fortune monitor or fortune teller: Understanding the connection between interaction patterns and financial status. In Privacy, security, risk and trust (passat), 2011 ieee third international conference on and 2011 ieee third international conference on social computing (socialcom), pages 200-207. IEEE, 2011.

[67] M. Pantic and L. J. Rothkrantz. Toward an affect-sensitive multimodal human-computer interaction. Proceedings of the IEEE, 91(9):1370-1390, 2003.

[68] A. S. Pentland. Automatic mapping and modeling of human networks. Physica A: Statistical Mechanics and its Applications, 378(1):59-67, 2007.

[69] A. S. Pentland. Honest signals. MIT press, 2010.

[70] E. J. Phares. Locus of control in personality. General Learning Press Morristown, NJ, 1976.

[71] F. Pianesi, N. Mana, A. Cappelletti, B. Lepri, and M. Zancanaro. Multimodal recognition of personality traits in socia interactions. In Proceedings of the 10th international conference on Multimodal interfaces, pages 53-60. ACM, 2008.

[72] O. Pierre-Yves. The production and recognition of emotions in speech: features and algorithms. International Journal of HumanComputer Studies, 59(1):157-183, 2003.

[73] H. Pirsiavash and D. Ramanan. Detecting activities of daily living in first-person camera views. In Computer Vision and Pattern Recognition (CVPR), 2012 IEEE Conference on, pages 2847-2854. IEEE, 2012.

[74] R. D. Putnam. Comunidade e democracia: a experiência da Itália moderna. FGV Editora, 2005.

[75] J. R. Quinlan. C4.5: Programs for Machine Learning. Morgan Kaufmann, 1993.

[76] B. Reeves and C. Nass. The Media Equation: How People Treat Computers, Television, and New Media Like Real People and Places. Cambridge University Press, New York, NY, USA, 1996.

[77] S. L. Robinson. Trust and breach of the psychological contract. Administrative science quarterly, pages 574-599, 1996.

[78] D. M. Rousseau, S. B. Sitkin, R. S. Burt, and C. Camerer. Not so different after all: A cross-discipline view of trust. Academy of management review, 23(3):393-404, 1998.

[79] B. Schneier. Liars and outliers: enabling the trust that society needs to thrive. Wiley. com, 2012.

[80] D. Schulman and T. W. Bickmore. Persuading users through counseling dialogue with a conversational agent. In PERSUASIVE, page 25, 2009.

[81] J. Shotton, T. Sharp, A. Kipman, A. Fitzgibbon, M. Finocchio, A. Blake, M. Cook, and R. Moore. Real-time human pose recognition in parts from single depth images. Communications of the ACM, 56(1):116-124, 2013.
[82] V. K. Singh, L. Freeman, B. Lepri, and A. S. Pentland. Classifying spending behavior using socio-mobile data. HUMAN 2(2):pp-99, 2013.

[83] J. Staiano, B. Lepri, N. Aharony, F. Pianesi, N. Sebe, and A. Pentland. Friends don't lie: inferring personality traits from social network structure. In Proceedings of the 2012 ACM Conference on Ubiquitous Computing, pages 321-330. ACM, 2012.

[84] C. Steinfield, N. B. Ellison, and C. Lampe. Social capital, selfesteem, and use of online social network sites: A longitudinal analysis. Journal of Applied Developmental Psychology, 29(6):434$445,2008$.

[85] J. Sturm, O. H. van Herwijnen, A. Eyck, and J. M. B. Terken Influencing social dynamics in meetings through a peripheral display. In ICMI, pages 263-270, 2007.

[86] K. Subrahmanyam, S. M. Reich, N. Waechter, and G. Espinoza. Online and offline social networks: Use of social networking sites by emerging adults. Journal of Applied Developmental Psychology, 29(6):420-433, 2008.

[87] E. A. Suma, B. Lange, A. Rizzo, D. M. Krum, and M. Bolas Faast: The flexible action and articulated skeleton toolkit. In Virtual Reality Conference (VR), 2011 IEEE, pages 247-248. IEEE, 2011.

[88] T. Toscos, A. M. Faber, S. An, and M. P. Gandhi. Chick clique: persuasive technology to motivate teenage girls to exercise. In CHI Extended Abstracts, pages 1873-1878, 2006.

[89] M. Turk and A. Pentland. Eigenfaces for recognition. Journal of cognitive neuroscience, 3(1):71-86, 1991.

[90] T. W. Valente. Social Networks and Health: Models, Methods, and Applications. Oxford University Press, 2010.

[91] T. W. Valente. Network interventions. Science, 337(6090):49-53, 2012.

[92] B. Waber. People analytics: How social sensing technology will transform business and what it tell us about the future of the work. Pearson Education, 2013.

[93] R. Y. Wang and J. Popović. Real-time hand-tracking with a color glove. In ACM Transactions on Graphics (TOG), volume 28, page 63. ACM, 2009.

[94] D. Wyatt, T. Choudhury, J. Bilmes, and J. A. Kitts. Inferring colocation and conversation networks from privacy-sensitive audio with implications for computational social science. $A C M$ Transactions on Intelligent Systems and Technology (TIST), 2(1):7, 2011.

[95] J. Yamato, J. Ohya, and K. Ishii. Recognizing human action in time-sequential images using hidden markov model. In Computer Vision and Pattern Recognition, 1992. Proceedings CVPR'92., 1992 IEEE Computer Society Conference on, pages 379-385. IEEE, 1992.

[96] M. Zancanaro, B. Lepri, and F. Pianesi. Automatic detection of group functional roles in face to face interactions. In Proceedings of the 8th international conference on Multimodal interfaces, pages 28-34. ACM, 2006. 